\title{
Modeling of the Universals of the Futsal: Exploration and Reading of the Game
}

\author{
Kacem Nejah¹, Naffeti Chokri², Guemriayman Elloumi Ali $^{3}$ \\ ${ }^{1}$ State, Culture and Change of Society, Higher Institute of Sport and Education Physics Ksar Saïd, Manouba, Tunisia \\ ${ }^{2}$ Group for the Study of Development and Social Environment (GEDES), Faculty of Human and Social Science of Tunis, \\ Tunis Tunisia \\ ${ }^{3}$ Educational Department of PAS, Higher Institute of Sport and Education Physics of Sfax, Sfax, Tunisia \\ Email: kacemnejah@gmail.com
}

How to cite this paper: Nejah, K., Chokri, N. and Ali, G.E. (2017) Modeling of the Universals of the Futsal: Exploration and Reading of the Game. Open Journal of Social Sciences, 5, 46-68.

https://doi.org/10.4236/jss.2017.511005

Received: October 9, 2017

Accepted: November 13, 2017

Published: November 16, 2017

Copyright ( $) 2017$ by authors and Scientific Research Publishing Inc. This work is licensed under the Creative Commons Attribution International License (CC BY 4.0).

http://creativecommons.org/licenses/by/4.0/

(c) (i) Open Access

\begin{abstract}
The highlighting of the deepest identity of Futsal is through putting into perspective the framework chosen for this new look, the driving praxeology, and the sports game is before all a body of rules. This playful contract, which is basically a social contract, determines for the most part, the internal logic that is specific to each game. The issue is to observe how the individual acting and deciding, immersed in this set of constraints, operates its practical choice and develops its own motor strategies. In short, this stability that characterizes all sports games reveals a crucial trait of their intelligibility. Besides, "a sports specialty was likely to appeal to a group only if its internal logic is consistent to the habitus of this group", emphasizes Parlebas (1981) [1]. This is to promote a new interpretation of sports. What seems to be mathematical or sociological studies companies on the game? The interest which was granted him does simply not honor. The major challenge will be to reconsider the sports game in terms of specificity which calls for original research and serious methodologies in a new light. Through time, we can realize that an avalanche of theories about the game appeared, each based on the "imperfections" of the other without however the solution she claimed to hold.
\end{abstract}

\section{Keywords}

Modeling, Universals, Reading of the Model, Futsal

\section{Introduction}

To set the sports game, it is essential, as a result of the work of Parlebas, nuance between two types of games that did not receive the same consideration on the 
part of the governing institutions. On the one hand, we find institutional sports games that have been awarded "order of merit" institutional. Preferential treatment was given and they were able to access the closed circle of official practices. The presence of national or international federations with all events arising therefrom is the perfect example. We fear example all team sports. On the other hand, we have the non-institutional sports games or traditional games which were abandoned to their fate and which did not have right to the benevolent attention of the authorities. Here we find the different games of our childhood: the ball to the prisoner, the ball sitting (Parlebas, 1986) [2]. This distinction between institutional and traditional game is capital and relates to pedagogical and sociological considerations. Called sports game, any driving situation of confrontation codified, referred to as "game" or "sport" by social bodies (Parlebas, 1999) [3]. Two distinctive criteria seem necessary in the first place: first test of driving situation that pits sports games to non-sports games (poker, chess...). Then, the coding that makes the game is basically defined by a system of rules that specifies the operating conditions. Sport can adopt this definition of the sports game but would add two decisive distinctive criteria, competition and institutionalization. On the first test, a physical practice cannot be regarded as a sport outside a competitive framework. It is precisely this context that makes a daily run or a simple swimming cannot be called "sport". The second criterion of institutionalization is very strong and returns to a capital distinction: sport, on the one hand, recognized and formalized (federation, Executive Board, technical Committee, referees, official texts...) and on the other hand, the rest of the fun practices. The game sports and all the "roughness" of the terrain involved are now not to apprehend without guarding a systematic observation and rigorous experimentation. Our goal is not to reduce the game to a simple methodological premise; the playful sense would lose for sure interest. Our purpose is to draw valuable lessons that we will avoid some unnecessary "mistakes" before pushing the analysis more far. The first point concerns the need to delimit the field of definition of the sports game in order not to fall into any landslides from field to field. The agreed definition exceeds looking for a Genesis to be based on identifiable and objectively quantifiable parameters.

\section{Theoretical Approach}

\subsection{Sport: An Institutional Distinction}

All sports were the basis of the games, other practices that games have been listed, but it's the institutional Prism, who spoke for only a limited part of the playful spectrum. As demonstrated by Parlebas P (1974) [4], practices analysis reveals that the choice of the institution focused on a particular batch of sports practices, values some playful contracts rather than others, favors certain types of competition (duel for example structure, search for the spectacular meetings). The highlighting of the underlying structures leads to a sociological observation: the sports institution pushed all models of complications and reversals of al- 
liances; she held that the purest duels between two welded teams without ambiguity. In definition, the institution chose the type of collective struggle the most basic possible. "The binary conflict promotes symbolic projections where opposing good and bad, those of my camp and others," observes Parlebas. Such a system promotes the projection on a meeting of performances of any kind: choice of a favorite, chauvinism, nationalism, but also the ideal of a fair fight between two masterfully opposing groups that grow at its highest level the social realization of man in its drive lines. A high symbolic function is represented by the sports show. One sure thing is that the head of the sport, presides over a system of rules very elaborate that no one may transgress on pain of being excluded or condemned.

\subsection{The Precedence of the Rules}

Many authors claim that the games are free and spontaneous activities. It is nothing of the kind. The basis of every sports game is a must in the first place a body of rules which determines the modalities of functioning of the game. In fact, the rules represent the fundamental matrix of locomotor acts. Play it is to submit voluntarily to a system of constraints. This is a paradox. Everything happens as if wanting to "break free" in the game, the man had introduced a guardianship of his freedom. As well as writing Parlebas, P (2010) [5]: "the freedom of the player only takes on meaning as seen in the context of a prior micro-contract". Presenting an obligation clause initially, this contract provides a note of satisfaction. We now find satisfaction in the constraint. If not, everyone will act in his own way and behave erratically and even insignificant. Indeed, the absence of duty would result, in the words of Raymond Boudon, "perverse" and a harmful dispersal of efforts. The irrational conduct is such that each would focus on a small personal profit which would be damaging for everyone and everyone they had to create a structure of interaction where the requirement would result in overall satisfaction. Agreed the code governs the game and invite everyone to get involved and to socialize. In establishing this, order is restored and the disorder abolished. However paradoxical, is that this agreement or agreement between participants is expressed through a fight (duel). We are cooperating to oppose. There-there-, from the relevant traits that underlies this beautiful universal agreement anyway? Indeed, all of the prescriptions issued by the code of the game seem to relate more to observable behaviors that watching this legitimate guardian, which is none other than the arbitrator. The untrained viewer more interested in this type of conduct apparent easily detectable and cares little about the overall shape, embracing the game and which is, let's face it, difficult to access. Can we reduce the game to simple behaviors of surface based on any logic? Can provide the deep structures underlying sports games?

\subsection{The Internal Logic}

The idea for our research that fits into the logic of the praxeology driving is that behind variable players, and legible lines prowl the universals. The goal is to 
move to another level of analysis. It is, in effect, to consider sports games from the point of view "inessentiel" of outward appearances (material used for the game, for example), but to approach them in terms of their relevance praxic: the internal logic driving lines. That is to say, the system of the relevant features of the locomotor situation and the practical consequences it entails. The notion of internal logic is in our eyes a cardinal in the sense that it is part of the identity of the game. The internal logic of any sports game comes foremost from its system of rules. For the mathematician Marc Barbut (1967) [6] "it is important to develop the point of view of the essential internal logic (codified generally games), as she emerged from the analysis of the rules of the game". Indeed, although the rule is first, it go unnoticed so that it is trivial. How to ignore such a system that largely determines the logic internal locomotor activities? It is this musculoskeletal system code that will make players intelligible and meaningful lines. For example, the futsal player internalizes, indeed, all of the requirements of the code and dictates them to his body when he plays it conveys to his body set, do not come into contact with the opponent under penalty of being sanctioned. Thus, the system of rules becomes a powerful carrier normative mediator of the internal logic of the driving situation and determines the behavior of the player through a habitus. These behavioral rules, imperatives are the hotlines. Not only has the locomotive contract sealed a constraint system that is largely responsible for body shaping but also the model of the game. Every game, it seems, has its own model based on his body of rules. Our goal is certainly not to propose a model for every sports game; this would be impossibility, but to identify the relevant traits that mark of inevitable way the structure of sports games. "It shows the structural invariance by the variance of the models", wrote Michel Serres. Each model particularly reports on a specific aspect. For example, the symmetric model, so prized by the institution and that always puts face-to-face two "equal" antagonists, makes account of the balance of the duel. Thus, when analyzing the structure of institutional games, one realizes that it is always this same model that arises every time, it is a permanent. Indeed, the graphs of the institutional games are balanced graphs. The mathematical representation takes therefore the significance and promotes the illustration that will allow us justified interpretations. "Behind the disorder of surface, seems to hide a deep order", writes Parlebas, the internal logic of the sports games. This powerful internal consistency is based on observable, measurable, and operating structures representing the bearing wall of the logic of the operation ludosportif and called universals. The study of universals of any sports game makes this game with its own identity and print players a motor behavior model distinguishing it from other models. Can we further deepen the notion of universals before undertaking the modeling of the sports games?

\subsection{The Notion of Universals}

The first objective of our thesis is to apprehend the futsal not a physiological or 
psychological point of view but to approach it in its praxic perspective the sports game with indeed fundamental praxic originality which is the driving action, must be able to analyze itself, and deserves not to be thinking to other fields that, interested not in the sports game but use it rather as related exploration object. It is on the side of the motor praxeology we must resolutely turn. Parlebas (1983)

[7] offer, in this context, a conceptual and praxic framework unique to a structural and methodological sports games approach. His theory of the driving action, called driving praxeology, posits the hypothesis according to which all sports games are preoriented by an internal logic in action or interaction systems. They rely on verifiable material: rules of the games and motor behaviours of the field. After observation and analysis, the methodological approach proposes to build the universals of the sports game, IE a way identifiable, underlying this model. This model has a capital share of the intelligibility of the game. The praxeologue is now "self-sufficient" and the theory of driving action seems finally him pave the way and provide the tools to protect themselves against the dictates of other disciplines and allow him to think of himself. Strictly observing the sports games, it will exceed the level of the surface of the motor behaviours to plunge into the world of deep structures that he'll try to objectify and quantify. What are these operating structures called universals that the praxeologue will seek to model. Model universals of a sports game, it's put in the open model of this game's reveal the relevant traits that characterize this game and bring out the internal logic of this game. The task is not as obvious as it sounds. It is, indeed, to operate a permanent observation and analysis requiring delicate treatment and complex materials deemed most relevant. But there is also a constant back and forth between the requirements of the rules and drive lines observed in the field. Without this "rough" path, our approach is more akin to metaphorical flights. In this perspective, the use of a mathematical representation is more beneficial insofar as it does not compromise. She refers to a validation supported assumptions and an armed confrontation models. But it means to consider the player as a passive bystander who is in a world of structures that are needed? According to the theory of sports games, there seems to be no contradiction between "the autonomy of the agents and the constraints of structures", borrowing the words of Raymond Boudon. Parlebas (1988) [8] will argue that it is precisely the constraint that gives the player his decision-making power, "by imposing part of the invariant structures, he wrote, the internal logic of the game gives rise to the infinite variations of the originality of the players and groups". One can think that far from any structural mechanization, the player full custody capacity initiative and decision-making within the constraints imposed by the rules of the game. Moreover, what we need to emphasize is that the modeling claims by no means exhaust all phenomena, claim to completeness would report more than a systems approach; however, there is not our goal. Our purpose is to report on the aspects considered relevant to the situation, which we'll try to connect. It's more than the static aspect of the game, is the locomotor dynamics that is proposed to show through its interaction mechanisms highlights Parlebas (2002) 
[9]. The project being asked, it seems necessary however to bring a background shade. The notion of universal may suggest that the sports game takes place on a fundamental invariants canvas which is combined outside a social context. The risk is real. It was already noted in other areas such as linguistics attend to universality or "universal laws" that would be ubiquitous across all languages. Thus, Roman Jacobson (1976) [11] asserts the existence of phonological universals or Chomsky (1969) [10], which did not hesitate to speak of "universal grammar". There was also the same tendency to consider certain human behaviors as identical regardless of their culture of belonging through remarks by Eibl-Eibesfeldt (1976) [12] who believes in universal behavioral consistency. Its bold design pushed him up to claim "human programmed". This orientation to the structuralization ran to fairy tales. Although Claude Levi-Strauss is considered to be the precursor to the structural approach, Propp in 1928 is already busy to extract Russian tales of stable structures.

In the field of games, Marc Barbut seems to have paved the way by suggesting a structural framework in which can now fit the coded games. For the case of sports games, it seems to hold the same cap. Contrary to what may think some, structural option does not reduce the locomotor parachuted a simple mathematical logic matrix. The sports game, before being a structure, is above all a social fact. For example, football can be seen as a society in miniature, as a laboratory of pipes and human communication (for example, the pass that is a technical base in football is a form of communication between partners). Even more, the presence of an institution at the head of this sports game, of an official text which governs, a legitimate guardian who ensures the application of rules, roles sociomoteurs, of a code of original, a duel of team communication, spectators... make football field favored for the observation of the dynamics driving socio, and more generally, for the study of the action. However, this structure is put into its framework. We can't uproot her. It's in its social perspective, the sports game to find its meaning. Outside his environment, it would look more to this silent nature Georges Hébert was talking. Cultural influences on the physical practices seem hardly questionable. "All education, writes Ulmann, devotes a cultural intervention of normative". The forerunner who put emphasis on the cultural dimension of 'techniques of the body, is without a doubt Marcel Mauss (1934) [13]. According to him, "body techniques" have that replongees sense in their social roots. All sports game would eventually be a reflection of its society of belonging and reflecting an ethno-playfulness. Down this road, Parlebas plans to undertake ethnology from the physical practices. The project is ambitious but could not jeopardize the notion of universals?

The deep analysis of sports allows two basic differences that can blow the homogeneity of universals. On the one hand, there is a remarkable intercultural variance where companies present very different locomotor activities content from each other. The approach, far from being ethnocentric, therefore comparative between the different practical content of their universals and makes it possible 
to short the original locomotor structures implementing. Indeed, several authors place the interaction between game and company at the heart of their problem. Marc Barbut opens the way by suggesting: "maybe the study of the structure of the games played by the companies will have then a role as revealing as those structures of kinship". It seems that the structural analysis of the sports games such as charged by the companies can identify certain standards and certain core values of the company concerned. One is led to think that the variances inter or intra cultural identified refuse not the notion of universal. The identified differences can, on the contrary, "validate each representative of universals", revealing the originality of such company or such group in its adoption of the model, and refer especially to the relevance of "overhanging meta-model". It would be interesting depending on Parlebas (1990) [14], universals can set "by their similarities and their differences, a larger global system: the system of sports games".

Modeling of universals of futsal is the major goal mapped out for this work. Therefore, find it us useful after you have defined the concept of place in the context of sports games

\subsubsection{Universals of Sports Games}

During a sports game, driving action of players has several meanings. First, the player in a very elaborate structure of exchanges communicates with its partners by friendly delivery of the ball, for example, and against communicates with opponents foiling their plans. Through this model of communication imposed by the rules of the game, we can already define a universal of prime importance: the driving communications network. Also, this communication is often not obvious. She can marry several forms. It can be sign language or praxic. The action to stand for example is a praxic communication consisting of one meaning (moving) which the meant is the call of the ball. Thus, participants must constantly decode behaviors to make signs. It happens so all coding of signs that can be represented by a second universal no less important than the first and that can be a very original: the semiotic code. Similarly, each player takes a role. For example, in football, unlike what you believe there not eleven roles but only two engines social roles: the goalie and field player. Indeed, each of them has a role that is well defined by the rule and delimited by the space reserved. The goalkeeper, out of its area, has more right to touch the ball with his hands. That's compared to his space, that the rule gives it the power to hold the ball with the hands, as football is a game of feet. However, the other ten players all have a same role, they can all defend attack, pass, shoot... but they cannot defend their cage with the hands, is it that the two roles are different. In addition, each role socio engine, can accomplish several tasks. For example, the fields in Handball player can dribble, pass, shoot... All of the motor tasks that are permitted by the rules of the game and that can accomplish the player are called motor socio subroles. Identify the network roles and the subroles can be illustrated through the establishment of the graph of the roles and one of the engines socio subroles. 
There are two other fundamental invariants. The fifth universal which is just as important is the system of scores. The institutional goal of any player from any collective playing field is to score points. It's the brand that is responsible for the change in the score. However, the mark cannot be attained by action of counter-communication such as fire in the opponent's "cage". That's who puts claims that the sport is a game of cooperation. The scores of the games institutional system is based on accounting and on time. The team that wins is the one that recorded the most points in time limits. This helps establish support all institutional games brand. This support allows us to formalize the possible path, as in a PLC, the evolution of the score. These universals are at the heart of the acts of game and can be represented by models of logical Mathematics type, in which pass necessarily the players and the game. However, the individual playing and acting, good intentions and decision, combines its drive lines on a frame which the waning is virtually predetermined preferences, precisely in the context of universals. In this perspective of modeling, apart from these universal essential to the derivative of the operating structures of the game, should be also given considerable importance to the concept of "game time". The hang of game stands as the unit of basic analysis of sports games in this sense, share its demarcation between two terminals definable, it allows to split the game in many discontinuous segments and is involved in the establishment of universals and the creation of the model specific to the game. For example, for the case of football, we'll hear per game move entire sequence between two stoppages. So that modeling is not possible in the purely speculative field of literary metaphors, we believe that the use of the constraints of the mathematical tool can be a great help. In the case of sports games, what can we expect this modeling? Are universals an asset in the search? Mathematical modeling has explanatory power?

\subsubsection{Properties of Models and Modeling}

A model is primarily relevant simplification of the situation at hand. It objectively represents all the range of possibilities that fit within the rules. The use of the graph theory allows obtaining such representation. "Power cleaner of some models by the mere fact of a formalized description of well chosen highlights full, on a goal mode, revealing elements usually watered down or hidden" Parlebas (2002) [15]. He must admit that models do not claim to be exhaustive nor exhaust the phenomenon. They represent only the relevant features of the situation. This simulation must be based on observable effects. It is in this state of mind that futsal modeling relies on which motor behaviors: passes, interception, dribbling, shooting at the basket... The model must also allow a formulation of a diagnosis and if possible a prognosis. The model can help understand the functioning of a social situation, or a sports game. It provides information on events that may occur; he anticipates that's its explanatory ability.

\subsubsection{Modeling of a Sports Game}

We present the main lessons learned from the modeling of an institutional game 
as illustration: volleyball. Now consider the model of volleyball. Its structure allows two types of interaction: on the one hand, driving communication of solidarity manifested here in the form of passes between teammates and on the other hand, the driving counter-communication of rivalry and which may in one against, lob, smash... Universal motor communications of volleyball can be summed up thus: between two players, there is never indifference: they are allies or opponents between two players, there is never ambivalent: they can be at the same time partners and opponents between two players, during the whole interaction is of a type unique, stable and always defined by rule. Continuing his work of modeling of the high competition volleyball, Parlebas (1988) was able to demonstrate, based on a comprehensive analysis of the 1986 World Championship matches, some "evidence" of volleyball was not as obvious. Indeed, as a result of a questionnaire sent to a sports population on the conduct of volleyball matches, results show that the percentage of errors in the answers is clearly high. This reflects a lag between conventional wisdom and the reality on the ground and a misperception of phenomena taking place yet in broad daylight: advantage of the maid, superiority of the attack team, lasting exchanges... The rigorous observation of the games was able to highlight the important role of service changes and was able to bring forth a new parameter in the analysis of games: the number of crossings of net characterizing each game calls and whose influence varies depending on whether it is even or odd.

By consulting the work of eminent experts on volleyball, was realized that this author explains the fickleness of the duration of the games by the imperative of the two points necessary to gain the set and the obligation to win three sets. The analysis of the conduct of the games showed that it is the change in service that is at the origin of this untimely duration of the parties.

Similarly, the counting of the results of the questionnaire, revealed that despite the presence of specialists (coaches and experienced volleyball players), average of incorrect answers exceeded that of the correct answers. For example, to the question: "volleyball team that is: much advantages, some advantages, advantages or disadvantaged, a little disadvantaged or significantly disadvantaged", $44.1 \%$ responded clearly have an advantage, then that modeling will allow us to assert the contrary.

\section{Methodology}

\subsection{Characteristics of the Study Population Observed}

In our approach, we worked on the collection of games from which we synthesize all possible motor interactions taking place during the game relating to the three levels of analysis. This function of collecting information on the object into consideration is based on an activity of coding done in continuous time (Table 1). During this operation we have followed the order of the actions of the players in interaction during the game. This procedure took about 4 minutes of observation for 7 seconds of games played. 
Table 1. Characteristics of the study observed population.

\begin{tabular}{ccc}
\hline Study population & Features & Number of matchs \\
\hline \multirow{2}{*}{ Professional Futsal } & World Cup 2012 & 8 matchs \\
\multirow{2}{*}{ Futsal club } & Europe Cup2012 & 8 matchs \\
& Tunisia 2012 Futsal Championship & 8 matchs \\
\hline
\end{tabular}

\subsection{Analysis of the Investigative Tool}

\subsubsection{Analysis of the Games: The Hang of Game}

In our modeling, they gave great importance to the concept of "game time". The hang of game stands as the unit of basic analysis of sports games in this sense, share its demarcation between two terminals definable, it allows to split the game in many discontinuous segments and is involved in the establishment of universals and the creation of the model specific to the game. For the case of Futsal, we defined the game as driving sequence shot between two stoppage time. Specifically, this sequence will extend from the point where the ball becomes "alive" until the moment when the ball becomes "dead". The definition of the hang of game in terms of "fenced" sequence occurs essentially in a methodological relevance perspective. So when is observed in a same time of game, several climbs hand ball and on the other can happen without any marked point, relevance lies in the fact should not be regarded as a landmark of analysis but rather the hang of game. Thus, not considered the hang of game like the action of a single team that ends his attack but rather driving interaction of the two teams which is in our opinion first, which gives the praxic unit its intelligibility. However, very often note in basketball of the phases where the whistle and the game "stops" to punish mistakes by some free throws. The regulation stipulates that during a free throw, the ball is considered to be alive. From there on, it is necessary to consider the free throw like a particular game insofar as although there is no actual interaction, the free throw shooting has relevance in the sense that it is a counter-communication that is very often involved in the change of the score Observation of the various games of Futsal could reveal some substantive differences in relation to sociocultural designs differing from the game and a fun code which is not the same for all the world Parlebas, (1998) [7] "the playful contract is basically a social contract" we can thus put the hypothesis that Futsal practice was not the same throughout the time she is not one who has begin with the practice of this sport from birth she is often inflected by the change in the rules of game. We suggest that by using verifiable methods such as the rules of the game and the motor behaviours on ground we can feel a game model for Futsal which determines the intelligibility of the game. It is from the rules of the game that we can grasp the meaning of the motor lines of players. Team sports induce inevitable way logic of cooperation between players. These perceptions are in a structure of Exchange or the player must cooperate with its partners to tackle an opponent. For this purpose we think that body of rules of Futsal has a methodological support based modeling: On the one hand on the observation of 
the matches as a reliable qualitative analysis tool i.e. a way identifiable underlying this model that determines the intelligibility of the game

\subsubsection{Statistical Procedure}

Feedback data, have been exploited by a data analysis software:

$\Leftarrow$ The average and standard deviation

$\Leftarrow$ Khi2 testing method: this is a statistical calculation that allows to decide the question of whether the relationship between two variables is significant or not. Specifically, it allows to test if two variables are dependent, in judging the significance of the differences between the theoretical results and the observed results. It compares an observed distribution (calculated from the formula of Khi2 (K2) applied on Excel) to a theoretical distribution, according to the table of distribution of Khi2 according to the degree of freedom (DOF) and the probability of permissible error. $\mathrm{DDL}=($ line -1$)($ column -1$)$

\section{Results}

We see based on the statistical results shown in Table 2; KHI2 is very significant at $P<0.001$, which is in favor of the two Futsals (the World Cup and $2012 \mathrm{Eu}-$ ropean Championship) which shows a significant difference at the level of the different variables of the motor translations during competitive situations of confrontation. Thus we retain first these difference very meanings in the use and the participation of the communications of the players during the game of the two Futsals (the World Cup and the European Championship) of the structures of their cultures and actions of collective interactions.

Table 2. The meaning of the motor lines from three different sociocultural contexts Futsal players adaptations to the regulation and internal logic.

Comparative analysis of motor translations during competitive situations of affrontement.des three Futsals according to the variation of the hang of the game

\begin{tabular}{|c|c|c|c|c|c|c|c|c|}
\hline & & $\begin{array}{c}\text { Average } \\
\text { number } \\
\text { of goals }\end{array}$ & $\begin{array}{l}\text { Average } \\
\text { number } \\
\text { of shots }\end{array}$ & $\begin{array}{l}\text { Average } \\
\text { number of } \\
\text { marking }\end{array}$ & $\begin{array}{c}\text { Average } \\
\text { number of } \\
\text { markdowns }\end{array}$ & $\begin{array}{l}\text { Average } \\
\text { number of } \\
\text { dribbles }\end{array}$ & $\begin{array}{l}\text { Average } \\
\text { number } \\
\text { of passes }\end{array}$ & Total \\
\hline \multirow{2}{*}{$\begin{array}{l}\text { C Monde } \\
2012\end{array}$} & Number & 47 & 100 & 19 & 6 & 12 & 6 & 190 \\
\hline & $\%$ & $24.0 \%$ & $51.0 \%$ & $9.7 \%$ & $3.1 \%$ & $6.1 \%$ & $3.1 \%$ & $97 \%$ \\
\hline \multirow{2}{*}{$\begin{array}{l}\text { C Europe } \\
2012\end{array}$} & Number & 46 & 110 & 12 & 5 & 9 & 4 & 186 \\
\hline & $\%$ & $24.2 \%$ & $57.9 \%$ & $6.3 \%$ & $2.6 \%$ & $4.7 \%$ & $2.1 \%$ & $98 \%$ \\
\hline \multirow{3}{*}{$\begin{array}{l}\text { C Tunisia } \\
2012\end{array}$} & Number & 36 & 80 & 6 & 2 & 2 & 2 & 128 \\
\hline & $\%$ & $27.7 \%$ & $61.5 \%$ & $4.6 \%$ & $1.5 \%$ & $1.5 \%$ & $1.5 \%$ & $98 \%$ \\
\hline & & \multicolumn{3}{|c|}{ Global } & \multicolumn{4}{|c|}{ Analytics } \\
\hline \multicolumn{2}{|c|}{ KHI2 } & \multicolumn{3}{|c|}{$882.5891^{* * *}$} & \multicolumn{4}{|c|}{8.5715} \\
\hline \multicolumn{2}{|c|}{ DDL } & \multicolumn{3}{|c|}{6} & \multicolumn{4}{|c|}{12} \\
\hline \multicolumn{2}{|c|}{$\mathrm{P}$} & \multicolumn{3}{|c|}{0.0000} & \multicolumn{4}{|c|}{0.7390} \\
\hline \multicolumn{2}{|c|}{ Decision } & \multicolumn{3}{|c|}{ T.S at $P<0.001$} & \multicolumn{4}{|c|}{ NS } \\
\hline
\end{tabular}


In collective actions, our two Futsals do not use so their functional strategies in this same vein note that values from watching the World Cup matches and the Championship of Europe define the middle of high-level competition pimp by a functional order mode group and individual, however, beyond the quantitative analysis a reading of arrays of condensation of the data (video) shows from the interactions that the definition of community developments in the individual and collective skills is relatively a factor organizational and participatory the precise definition of the elements of the environment within the meaning of Brousseau, 1990 [16].

The results achieved so far, allow us an investment of the space of the game by interacting with the physical qualities of players who determine an active and functional of each team model characterizes the fertile operation of our two Futsals.

In this respect two observations are needed:

First as a mode of collective organization allowing an investment game in interaction space determined by cultural factors of social references for each teams and this reflection differences found in the order of use of motor translations during competitive situations of confrontation.

The second concerns collective organization allowing an investment of the space of the game by interacting with the physical qualities of the players determining a model active and functional of each team characterizes the fertile feature Futsal.

The exception is the significant difference for the different variables to intersect with the results of observation of the Championship of Tunisia. This during the significance test shows that the individual technique seems to be in favor of the meaning of a successful individual or collective to decode an organization of the opponent. To better understand changes in motor translations during competitive recall skills are a result of cooperation by calling a confrontation with the other skills during the responsibilities of the performance of the tasks in situations of confrontation and are not in the same way it determines the effectiveness of teams and reflected in the scoring system to determine a winner for each competition. Also, it uses mainly so differentially individuals to act in a problem situation and overcome a barrier technical and psychological, the middle and the sharing of responsibility to situations. These findings brings us to present the order of meaning through the use of percentages that prove that the minimum of the percentages to the levels of the variables are related to the Championship of Tunisia in relation to the translations of the motor during the competitive of the three Futsals confrontation situations and therefore the identification of a bad management of the driving motor of the players who have move towards individualization of the game without the acquisitions drive and collective investment Table 2.

Figure 1 shows the representation of changes in driving translations during competitive situations of confrontation of the three Futsals in terms of percentages for the different variables, where we find after decortications of the results 


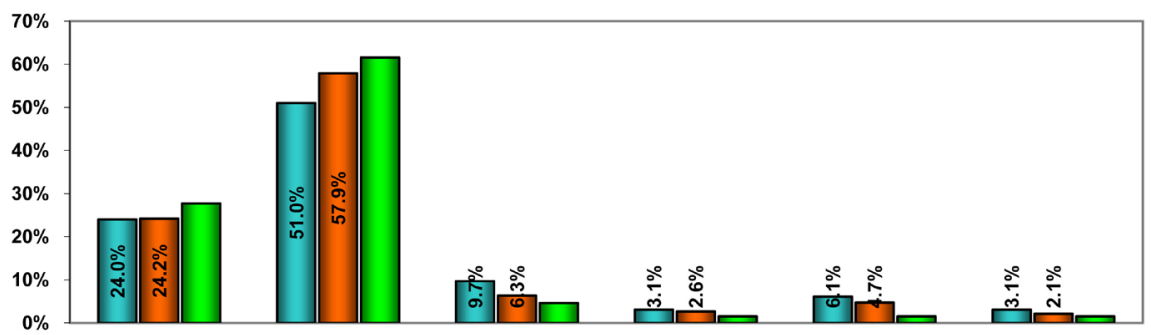

Figure 1. Graphical representation of comparisons of driving translations during competitive situations of affrontement.des three Futsals according to the variation of the hang of the game.

of this stage that:

- A difference very meanings in the use and the participation of the communications of the players during the game of the two Futsals (the World Cup and the European Championship) who do not use their functional strategies as well.

- The Futsal at the level of the World Cup is a game based on individualities.

- The different variables used in situations of confrontation are not in the same way it determines the effectiveness of teams and reflected in the scoring system to determine a winner for each competition.

- The Championship of Tunisia is identified by the no investment acquisitions and driving translations during competitive situations of confrontation. Study of the meaning of the motor lines from three different sociocultural contexts Futsal players adaptations to the regulation and internal logic. A development is important for analytical treatment of the variables of the meaning of Futsal players drive lines from three different sociocultural contexts emerged from the comparison of the adaptations with the regulations and the internal logic of this sport at the competitive situations of confrontation, thus from statistical values of the variation of these variables can our study population comparisons are reflected in Table 3.

The khi2 analysis on the meaning of the motor lines from three sociocultural contexts Futsal players, shown in Table 3 reveals a global khi2 $=2130,5737$ very significant at $P<0.001$. This shows that the three Futsals have made significantly different results. Statistical analysis shows that values from the observation of the European Championship and the World Cup games define the middle of high-level competition pimp by a functional mode of order, individual and collective, that while the percentage of variables of the meaning of the motor lines of players varied according to the cultural contexts as a source of development of the behaviour of the players in situations of confrontation, these values are in favor of the two Futsals (the World Cup and the European Championship) with values respectively at the level for example no respect for the rule of 5 meters (3.7\% World Cup \&gt; europe5 Cup, 5\% \&gt; Championship of Tunisia 9.5\%) these findings are identical for the different variables and whose object the study of the meaning of the motor lines (no respect of 4 second rule and no respect for the rules of the goalkeeper, penaltys of 10 meters). 
Table 3. The meaning of the motor lines from three different sociocultural contexts Futsal players adaptations to the regulation and internal logic.

\begin{tabular}{|c|c|c|c|c|c|c|c|}
\hline \multicolumn{8}{|c|}{$\begin{array}{l}\text { Study of the meaning of the motor lines from three different sociocultural } \\
\text { contexts Futsal players adaptations to the regulation and internal logic }\end{array}$} \\
\hline & & $\begin{array}{l}\text { Kick } \\
\text { game }\end{array}$ & $\begin{array}{l}\text { No Respect } \\
\text { of rule } \\
4 \text { second }\end{array}$ & $\begin{array}{l}\text { No respect } \\
\text { for the rule } \\
\text { of } 5 \text { meters }\end{array}$ & $\begin{array}{c}\text { Non-respect } \\
\text { of the rules } \\
\text { of the } \\
\text { goalkeeper }\end{array}$ & $\begin{array}{c}\text { Penalties } \\
\text { of } 10 \\
\text { meters }\end{array}$ & Total \\
\hline C Monde & Nombre & 240 & 15 & 10 & 2 & 2 & 269 \\
\hline 2012 & $\%$ & $89.2 \%$ & $5.6 \%$ & $3.7 \%$ & $0.7 \%$ & $0.7 \%$ & $100 \%$ \\
\hline C Europe & Nombre & 201 & 15 & 13 & 4 & 4 & 237 \\
\hline 2012 & $\%$ & $84.8 \%$ & $6.3 \%$ & $5.5 \%$ & $1.7 \%$ & $1.7 \%$ & $100 \%$ \\
\hline C Tunisie & Nombre & 290 & 27 & 37 & 18 & 18 & 390 \\
\hline 2012 & $\%$ & $74.4 \%$ & $6.9 \%$ & $9.5 \%$ & $4.6 \%$ & $4.6 \%$ & $100 \%$ \\
\hline \multicolumn{3}{|c|}{ Global } & \multicolumn{5}{|c|}{ Analytique } \\
\hline KHI2 & \multicolumn{2}{|l|}{$2130.5737^{\star * *}$} & \multicolumn{5}{|c|}{$31.9397^{\star * *}$} \\
\hline DDL & \multicolumn{2}{|l|}{4} & \multicolumn{5}{|c|}{8} \\
\hline $\mathrm{P}$ & \multicolumn{2}{|l|}{0.0000} & \multicolumn{5}{|c|}{0.0001} \\
\hline Decision & \multicolumn{2}{|l|}{ T.S at $P<0.001$} & \multicolumn{5}{|c|}{ T.S at $P<0.001$} \\
\hline
\end{tabular}

It also reflects a different reading of the rules, IE a greater flexibility in the application of the regulation and therefore a more fault tolerance. This is what is meant by cultural differences in individual and collective practice of the Futsal.et one set apart from technical qualities and all the factors of adaptations with the internal logic of the discipline.

Figure 2 shows the representation of changes in the significance relationships between the Futsals points of view adaptations with the internal logic in situations of competition apply and interacting with the functional mode of discipline which explains the meaning of individual or collective success that is dependent on motor lines of players in competitive situations, remember that this adaptation is a result of cultural development which calls for a social experience and is not only the nature of the competition and distribution of tasks and responsibilities. In the same vein that we retain for comparisons of the European Championship and cup of the world $p$ approaches to 0 , so the difference is very significant.

The results are reversed when it comes now to the comparison with the Championship of Futsal in Tunisia. Remember that the variations of the adaptations with the internal logic to ensure by the respect rules and drive lines of players can be one of the parameters of the dimension of valuations of the effectiveness of team addressing the elements of the environment in the individual and collective skills interactions and adaptations with the rules of the game.

Indeed most of the parameters that are listed in the following table are stable and present data, so close they can reinforce the notion of universals. 


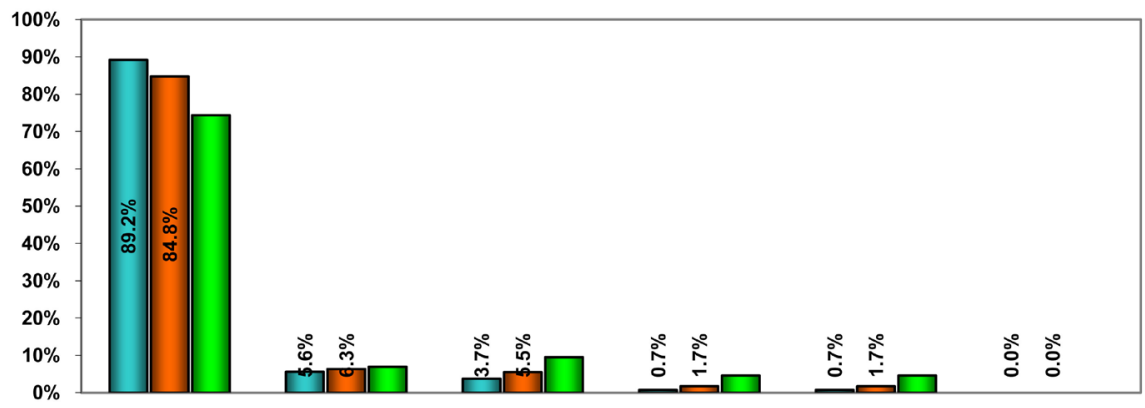

Figure 2. Graphical representation of the meaning of the motor lines from three different sociocultural contexts Futsal players adaptations to the regulation and internal logic.

In the pursuit of the objective that we have drawn from the start to explore more tracks we offer modeling to establish the structure or model of the current operation of Futsals of high competition matches (Figure 3). Playback of the observed data and the analysis of the results of the last of the world and Europe championships (2012) show that most of the parameters that are listed in the following table are stable and present data, so close they can reinforce the notion of universals (Figure 4).

The game of a high level of Futsal match current model has averaged 220 separate motor sequences with a duration of 5 seconds each. According to the observed model, can be found in the hang of game 5 assists, 10 dribbles, 1 markdowns and al shot. The values for each trait are represented in percentage in what follows (Figure 5).

In, first, of the body of rules of Futsal, we have established the structural analysis of the game to set the type of the style game Futsal representing each of the relevant traits (universals). And every universal may lend itself to any kind of interpretation. For example, the drive communications network tells us that game of Futsal model is mainly based on a structure of type symmetrical duel of team (Table 4).

The analysis of data and the expert consultation shows that there is clearly more duels one on one by match (Table 5). This allows us to think that this is the form that probably arises as Futsal.

Indeed, starting with the number of game by game (Table 6). We noticed that the two Futsals on this universal data converge near median value (220). This is the number of intermittent motor sequences observed in the game in a repetitive fashion model. The duration of these sequences of game turns, in turn, be a constant relevant feature, which strengthens the structure of the model. The values of the two Futsals, on this trait, not disparate, appear to be in perfect harmony. Thus, 5 seconds, such is average Futsal game shots before that any stoppage of play or a goal scored. The large number of passes per game call is probably a very necessary collective approach as driving solution in the face of opposing aggressive defenses and especially as essential means to evolve on the ground (Table 7).

The meaning of the motor lines of players from Futsal to three different 


\section{The 2012 World Cup Futsal}

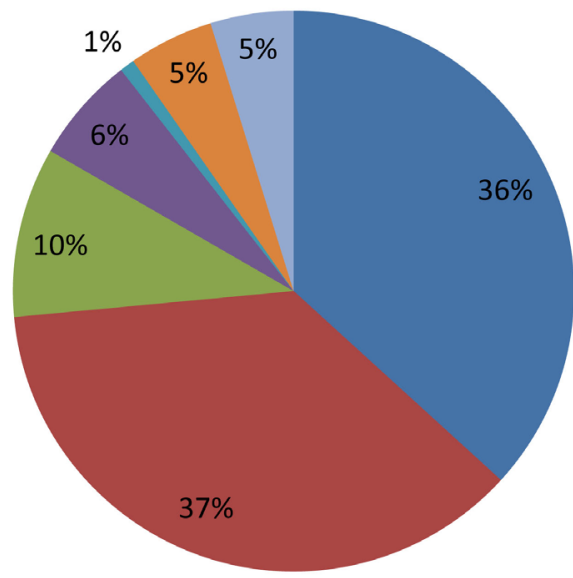

Duration of game call

number of Passes per game call

Number of markdowns. By stroke of game

Number of shots per game call

number of $10 \mathrm{~m}$ per blow of game Penalty

Value of game calls

percentage of the shots of game successful

Figure 3. The stable features of games model Futsals of the 2012 World Cup game.

Table 4. Table of stable features of Futsalsgames: The current model of game of Futsal.

\begin{tabular}{|c|c|c|c|c|c|c|c|c|}
\hline \multicolumn{9}{|c|}{ Modèle N1 } \\
\hline & $\begin{array}{c}\text { Number } \\
\text { of shot game }\end{array}$ & $\begin{array}{c}\text { Duration of } \\
\text { game call }\end{array}$ & $\begin{array}{c}\text { Number } \\
\text { of Passes per } \\
\text { game call }\end{array}$ & $\begin{array}{c}\text { Number of } \\
\text { markdowns. By } \\
\text { stroke of game }\end{array}$ & $\begin{array}{l}\text { Number of } \\
\text { shots per } \\
\text { game call }\end{array}$ & $\begin{array}{c}\text { Number of } \\
10 \text { m per blow of } \\
\text { game Penalty }\end{array}$ & $\begin{array}{c}\text { Value } \\
\text { of game calls }\end{array}$ & $\begin{array}{l}\text { Percentage of the } \\
\text { shots of game } \\
\text { successful }\end{array}$ \\
\hline The 2012 & & & & & & & & \\
\hline $\begin{array}{c}\text { World Cup } \\
\text { Futsal }\end{array}$ & 240 & 6 & 6 & 1.6 & 1 & 0.14 & 0.8 & $78 \%$ \\
\hline $\begin{array}{c}\text { The cup of } \\
\text { Europe } 2012 \\
\text { Futsal }\end{array}$ & 201 & 5 & 4 & 1.5 & 1.1 & 0.15 & 0.6 & $67 \%$ \\
\hline $\begin{array}{l}\text { model of } \\
\text { game (1) }\end{array}$ & 220.5 & $5.5^{\prime \prime}$ & 5 & 1.55 & 1.05 & 0.145 & 0.7 & $72.50 \%$ \\
\hline
\end{tabular}

Table 5. Table of stable features of model Futsals of the European game games.

\begin{tabular}{|c|c|c|c|c|c|c|c|c|}
\hline & $\begin{array}{l}\text { Number of } \\
\text { shot game }\end{array}$ & $\begin{array}{c}\text { Duration of } \\
\text { game call }\end{array}$ & $\begin{array}{l}\text { Number of } \\
\text { Passes per } \\
\text { game call }\end{array}$ & $\begin{array}{l}\text { Number of mark- } \\
\text { downs. By stroke of } \\
\text { game }\end{array}$ & $\begin{array}{l}\text { Number of } \\
\text { shots per } \\
\text { game call }\end{array}$ & $\begin{array}{l}\text { Number of } 10 \\
\text { m per blow of } \\
\text { game Penalty }\end{array}$ & $\begin{array}{l}\text { Value of } \\
\text { game call }\end{array}$ & $\begin{array}{l}\text { Percentage } \\
\text { of the shots } \\
\text { of game } \\
\text { successful }\end{array}$ \\
\hline $\begin{array}{l}\text { The cup of } \\
\text { Europe } \\
2012 \text { Futsal }\end{array}$ & 201 & 5 & 4 & 1,5 & 1,1 & 0,15 & 0,6 & $67 \%$ \\
\hline Model (1) & 220.5 & $5.5^{\prime \prime}$ & 5 & 1.55 & 1.05 & 0.145 & 0.7 & $72.50 \%$ \\
\hline
\end{tabular}

Table 6. Table of stable features of model Futsals of the high-level game games.

\begin{tabular}{ccccccccc}
\hline & $\begin{array}{c}\text { Number of } \\
\text { shot game }\end{array}$ & $\begin{array}{c}\text { Duration of } \\
\text { game call }\end{array}$ & $\begin{array}{c}\text { Number of } \\
\text { Passes } \\
\text { per game call }\end{array}$ & $\begin{array}{c}\text { Number of } \\
\text { markdowns. } \\
\text { By stroke of game }\end{array}$ & $\begin{array}{c}\text { Number } \\
\text { of shots } \\
\text { per game call }\end{array}$ & $\begin{array}{c}\text { Number of } 10 \mathrm{~m} \\
\text { per blow of } \\
\text { game Penalty }\end{array}$ & $\begin{array}{c}\text { Value of } \\
\text { game calls }\end{array}$ & $\begin{array}{c}\text { Percentage of } \\
\text { the shots of } \\
\text { game successful }\end{array}$ \\
\hline $\begin{array}{c}\text { Reference } \\
\text { model }\end{array}$ & 220.5 & 5.5 & 5 & 1.55 & 1.05 & 0.145 & 0.7 & $72.50 \%$ \\
\hline
\end{tabular}


Table 7. Table of stable features of the model Futsals of the Tunisia game games.

\begin{tabular}{|c|c|c|c|c|c|c|c|c|}
\hline & $\begin{array}{l}\text { Number of } \\
\text { shot game }\end{array}$ & $\begin{array}{c}\text { Duration of } \\
\text { game call }\end{array}$ & $\begin{array}{l}\text { Number of } \\
\text { Passes per } \\
\text { game call }\end{array}$ & $\begin{array}{c}\text { Number of } \\
\text { markdowns. } \\
\text { By stroke of game }\end{array}$ & $\begin{array}{l}\text { Number of } \\
\text { shots per } \\
\text { game call }\end{array}$ & $\begin{array}{l}\text { Number of } 10 \mathrm{~m} \\
\text { per blow of } \\
\text { game Penalty }\end{array}$ & $\begin{array}{c}\text { Value of } \\
\text { game calls }\end{array}$ & $\begin{array}{c}\text { Percentage } \\
\text { of the shots of } \\
\text { game successful }\end{array}$ \\
\hline Model of the Futsal & & & & & & & & \\
\hline $\begin{array}{l}\text { Championship of } \\
\text { Tunisia in Futsal } 2012\end{array}$ & 290 & $3.075^{\prime \prime}$ & 2.44 & 0.7 & 0.816 & 1.08 & 0.4 & $42.50 \%$ \\
\hline
\end{tabular}

\section{The cup of Europe 2012 Futsal}

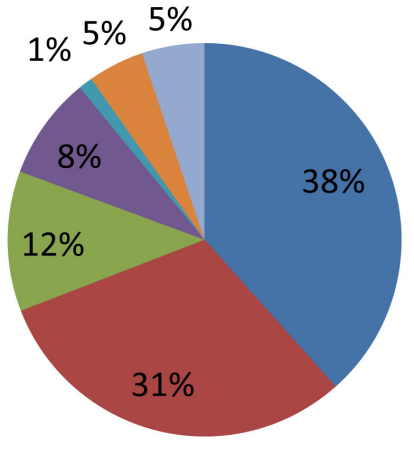

Duration of game call

Number of Passes per game call

Number of markdowns. By stroke of game

Number of shots per game call

number of $10 \mathrm{~m}$ per blow of game Penalty

- Value of game calls

percentage of the shots of game successful

Figure 4. The stable features of the model Futsals of the game of the 2012 European Cup matches.

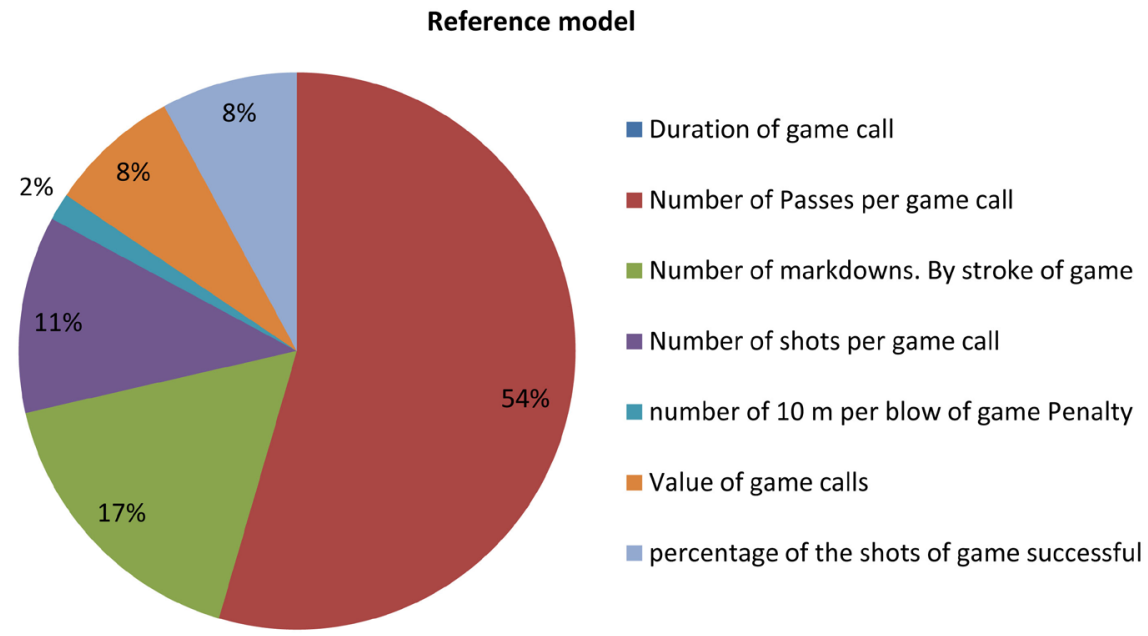

Figure 5. The stable features of model Futsals of the high-level game games.

sociocultural contexts emerged from the comparison of motor translations during competitive situations of confrontation reflecting a model from references the stabilities and the universals directs us based on the analytical results of the values chosen for culture Futsal in Tunisia (Figure 6).

\section{Discussion}

The reading of the game model depends largely of angle of attack from which it captures the element or the model. Indeed, in our analytical work, we approached the modeling from three different perspectives. We started by modeling by 


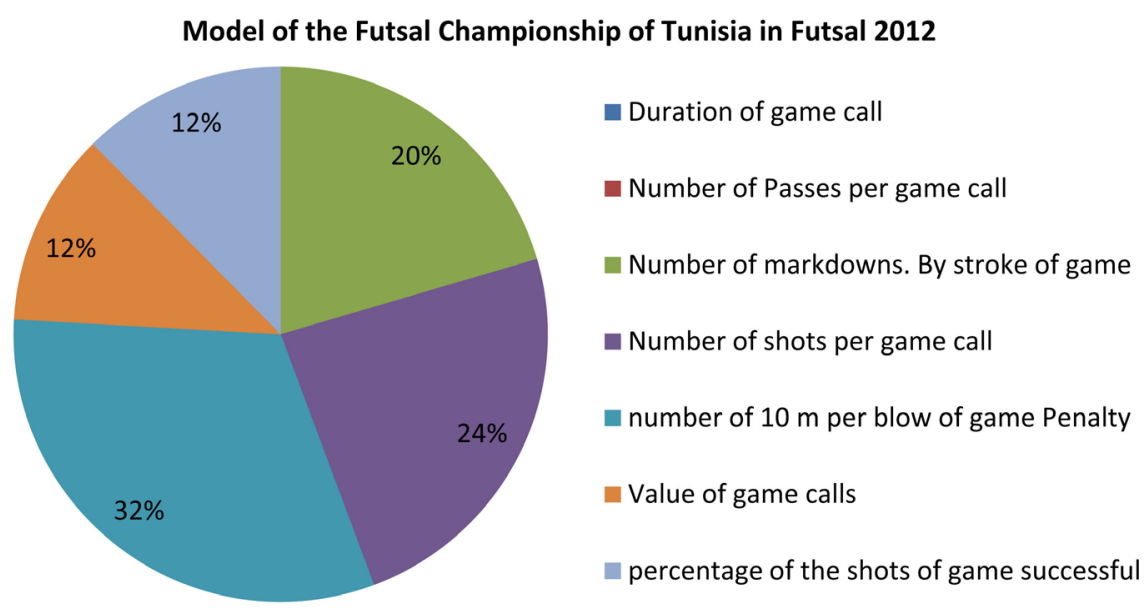

Figure 6. The stable features of games Futsals model of the game in Tunisia.

stroke play, match, and competitions. Each type of modeling data are not necessarily the same issues of the same internal logic of the game though.

By choosing the competition from which we want to take a look on the model or a model element, we can get reliable marks on the main composition of the considered system.

Thus, in considering, for example, modeling by stroke of game, one realizes that the game can be schematized as a combination of traits that make up the most relevant parameters for the definition of the hang of game. Indeed, it has been observed that the dribble and pass, for example, a very important position in the hang of game.

This representation seems probably simplistic and very simplified. Yet, she tells us more about phenomena repeating themselves stably suddenly game to another, and had observed a significant number of shots of game to reach such finding. Affirm that the pass and dribbling associated with shooting from the central core of a game call, we inevitably refers to basic findings which the content still occupies a good part of the workouts. Everyone agrees today that the fundamental basis of Futsal.

Moreover, the importance attributed to each line either in percentage terms or in terms of value, set apart from its significance on the game, may be subject to a sociological interpretation. Indeed, the predominance of the passes in the game, for example, us calls and refers us to another type of reading. Apart from its use to advance the field, we know that the past is above all a way to overcome the opponent, a tool to enter a duel with him, and this is the outcome of this duel will generally depend on largely as a result of the attack (fixing of the opponent).

One can think that the massive use of a technical base gesture that is the past, partially finds its meaning in the expression of the duel and in the way of "against-communicate" with the opponent. It is now acknowledged by analysts of the Futsal defensive systems implemented are rendered more and more aggressive so that passes and dribbling turns out are an effective way to break through the defense. The attack also seems to take precedence over the defense 
because defensive help is more difficult because of the risk of has errors and discrepancies caused by the defensive movements. Accordingly, it is said that there is a very marked increase in duels one on one essential element of fixing by dribbling facilitated also by markdowns but also by the crucial role of free players responsible above all to set the defense to liberate other players.

Indeed, the duel is not the attack but rather the defensive pressure on the attackers to prevent them from scoring. In other words it's defensive aggressiveness that will determine the outcome of the duel. It is probably a paradox that rather than focus on offensive engagement.

It seems indeed that the Futsal, promotes this model of game based essentially on the duel, reflecting the interests of antagonists for which oppose two opponents which supports more findings previously found during the reading of the rules. Where you can point out not only the form of duel emerges clearly in the structure of the game of Futsal model but through a staging, supported by logic of realization. We are faced with a situation of duel and show that are part of the institutional logic.

Our objective of departure for this work was to identify a model of game of Futsal based on relevant traits that reflect the internal logic of the game. To do this, we equipped our work reliable tools, on the one hand the body of Futsal rules and on the other hand, the observation of 16 matches of high competition. For this purpose, we had the hang of game as a unit of basic analysis in the sense that it allows to split the game into discontinuous segments. The results obtained allowed us to model some aspects of the functioning of the game (duration of the game footage, the traits that characterize). To apply for a finding of Futsal, he had to first trace its origins and track all the changes that the Futsal have known during its evolution to today. We then found that some rules such as the goalkeeper, contributed fully to the outbreak of the game. We therefore applied the hypothesis that the adaptation with Futsal rules would be operated mainly in terms of intimacy. In order to test this hypothesis, we have comparison of two Futsals. Indeed, we face the results of the matches of the World Cup 2012 where they practiced the rule of the goalkeeper. The results reveal interesting findings particularly with regard to the number, the duration of the hang of game. In taking note of changes in the current world Futsal, we decided to confront him, in turn; all equal proportion to an another Futsal playing the same rules but also has a great reputation, professional Futsal in Europe. By the way, we wanted to test the hypothesis according to which the practice of Futsal from a different culture would induce different logics and put at the same time the test of review the ideas that the world Futsal presents a form of gambling that is superior to the other Futsals.

Indeed, we have operated a second comparative analysis across the traits selected for modeling. The analysis reveals that the results of some parameters will not only against received ideas but discriminate between the Futsals. For example, it was shown you score fewer goals per game at the Championships, that whistle fewer mistakes and therefore we shoot at the penalty of $10 \mathrm{~m}$ or less, 
most of the features data show no significant difference between the two Futsals reinforcing the notion of universals. These traits are elements of structure in the creation of the game model. It seems that the Futsal today contains stable as dashes, for example, a match to 220 shots of game with a duration of 5 seconds. This game has a value and a chance to be successful. Also, in accordingly, first, of the body of rules of Futsal, we could establish the structural analysis of the game to set the type of the style game Futsal representing each of the relevant traits (universals). And every universal may lend itself to any kind of interpretation. For example, the drive communications network tells us that game of Futsal model is mainly based on a structure of type symmetrical duel of team. The analysis of data and the expert consultation shows that there is clearly more duels one on one by match. This allows us to think that this is the form that probably arises as Futsal. To close one can say that a Futsal match contains a total of functional and relational component determining a Co-construction of addressing a collective deciphering of the code of organization of the opponent during a phase of cons

The meaning of the motor lines of players from Futsal to three different sociocultural contexts emerged from the comparison of motor translations during competitive situations of confrontation reflecting a model from references to the stabilities and universal points based on the results of analysis of the values used for Futsal in Tunisia culture interested in the integration of this discipline in physical education and sports programming Indeed this sport which has started more than ten years in Tunisia make differences of meanings and adaptations with its own content and its internal logic so how can ensure social reproduction of form of this culture through teaching content?

\section{Conclusions}

Examination of the data of this comparative approach shows that most considered traits have not emptied significant divergences in the practice of Futsal. For example, we see that the percentage of hits of game pass per game or even the value of the hang of game is practically the same as it comes to high-level competition. It is, however, important to note that three variables were able to distinguish the Futsals. For example, the number of points scored per game or even the number of penalty of $10 \mathrm{~m}$ per match. These findings from three Futsals of origin and divergent culture (Europe, Africa and the World Championship), take us back, in effect, to focus more stable relevant features in the construction of the model of game. This new construction exceeds now the framework of differences by integrating all in a larger system which certainly reduces the disparities but which allows a new reading of the data. Indeed, the pursuit of the objective that we estimated to have modeling in order to establish the structure or model of the current operation of high competition Futsal matches.

Regarding the concept of modeling, it promotes a new interpretation of sports to clear mathematical or sociological studies companies on the game. The major challenge will be to reconsider a new sports game in terms of specificity, which 
calls for original research and serious methodologies. This key concept is at the center of our research at the base of every sports game, necessary in the first place a body of rules which determines the modalities of functioning of the game. Futsal rules represent, in fact, the fundamental matrix of locomotor acts. Play it is to submit full sound to a system of constraints.

Our results are consistent with the work of Parlebas (1998) [15] that signals in the first place make modeling happen through universals that define internal logic to the sports game. Second, the analysis of universals aims at switching "prescriptions based on the action of the agent deciding structures of interaction making intelligible the decision-phenomenon". So, the Futsal presents itself in a unique and identifiable configuration from the universals as illustrated in our modeling. The prime concern of gain, the team located in the obligation to cooperate and to oppose; it fits well in a structure of interaction by adhering to a system of constraints defined in the rules of the game. The game codes present an assortment of variable behaviors which it is necessary to identify the relevant traits. In other words, he comes to understand the mode of operation of the teams, by illustrating how communications and against communications to reveal the relationships that are established during the game sportif.ces findings which corroborate with our interpretation of the Futsal to understand the specificity of this sporting culture and the cultural representations out of the sport. Confirm by the thought of Georges Hébert that cultural influence on the physical practices seem hardly questionable. "All education, wrote Ullmann, devotes a cultural intervention of normative". Similarly, the forerunner who puts emphasis on the cultural dimension of "techniques of the body", is without a doubt through Marcel Mauss (1934) [16], which suggests the importance of body techniques that have meaning that replongees in their social roots. All sports game would eventually be a reflection of its society of belonging and reflecting an ethno-playfulness. Thus, our study joined structural analysis of that charged by the companies such as Futsal allows to detect certain standards and certain core values of the company concerned. One is led to think that the variances inter or intra cultural identified refuse not the notion of universal. The identified differences can, on the contrary, "validate each representative of universals", revealing the originality of such company or such group in its adoption of the model

So, the Determination of the effectiveness of the teaching of Futsal is revealed on effective practice being so practice exercised on a learner having favorable characteristics in terms of learning such as the levels of knowledge, the degree of motivation and the high social and cultural capital. Research of the effectiveness of the teaching was born with the challenge of the observation of the teacher's action is institutional directive highlighting the nature of the discipline and the internal logic of the game to understand the difference in performance between the various component of educational structures centered on the epistemological interaction of knowledge and skills, this finding of educational view of the system in relation to the company guiding has a concept of modeling the effectiveness in teaching which the goal is to estimate relationships between students and 
their products taking into account their socio-economic environment Momk (1992) [17], models of efficiency is a guide to alternation interactive of the determinants of learning, centered on a "micro" level which is the class. The quality of teaching is one speech in him speech psychological motivation of the lights the intelligence of the learner. Research efficiency measure on a performance criterion is confronted in their assessment of the overall pedagogical action by a structural hierarchy of base marked by the choice of the moment.

\section{References}

[1] Parlebas, P. (1981) Contribution to a Lexicon Commented in Science of the Motor Action. INSEP, Paris.

[2] Parlebas, P. (1986) Eléments de sociologie du sport. [Elements of Sport Sociology.] PUF, Paris.

[3] Parlebas, P. (1999) Games, Sports and Societies. Lexicon of Driving Praxeology, INSEP Publications, Paris.

[4] Parlebas, P. (1974) Elementary Mathematical Analysis of a Sports Game. Mathematics and Social Sciences, 47, 5-35.

[5] Parlebas, P. (2010) Mathematics and Social Sciences. pp.33-50.

[6] Barbut, M. (1967) Games and Mathematics. Gallimard, Paris, 836-864.

[7] Parlebas, P. (1983) Sports and Games. Special to New Education, Cemea.

[8] Parlebas, P. (1988). Analysis and modeling of volleyball of high competition. In Science and traction, no. 4, pp. 3-22. Paris: INSEP.

[9] Parlebas, P. (2002) Elementary Mathematical Modelization of Games and Sports. In: The Explanatory Power of Models, Bridging the Gap between Empirical and Theoretical Research in the Social Sciences, Metodos Series, Kluwer Academic Publishers, Dordrecht, 197-227. https://doi.org/10.1007/978-1-4020-4676-6_11

[10] Chomsky (1969) America and Its New Mandarins. Editions du Seuil.

[11] Roman Jacobson (1976). https://www.amazon.fr/Roman-Jakobson/e/B001ILHL7K

[12] Eibl-Eibesfeldt (1976) Human Programmed. The Innate, Determinant of Human Behavior (Der Vorprogrammierte Mensch, 1973), transl., Flammarion, 1976, 256 p.

[13] Mauss, M. (1934) Body Techniques. Article Originally Published Journal of Psychology, XXXII, ne, 3-4, 15 March-15 April 1936. Paper presented at the society of Psychology on 17 May 1934. http://www.regine-detambel.com/images/30/revue_1844.pdf

[14] Parlebas, P. (1990) Physical Activity and Motor Training. EPS Editions, Paris. (1st edition: 1976).

[15] Parlebas, P. (1998) Games, Sports and Societies. INSEP, Paris.

[16] Margolinas, C. (1998) The Middle and the Contract, Concepts for the Construction and Analysis of Situations. https://Hal.Archives-Ouvertes.Fr/Halshs-00421845/Document

[17] Jorion, P. (1986) Bayes-Stein Estimation for Portfolio Analysis. The Journal of Financial and Quantitative Analysis, 21, 279-292. https://doi.org/10.2307/2331042 


\section{Annexes}

Grid observation of the Futsal

Match:

Event:

Result: .

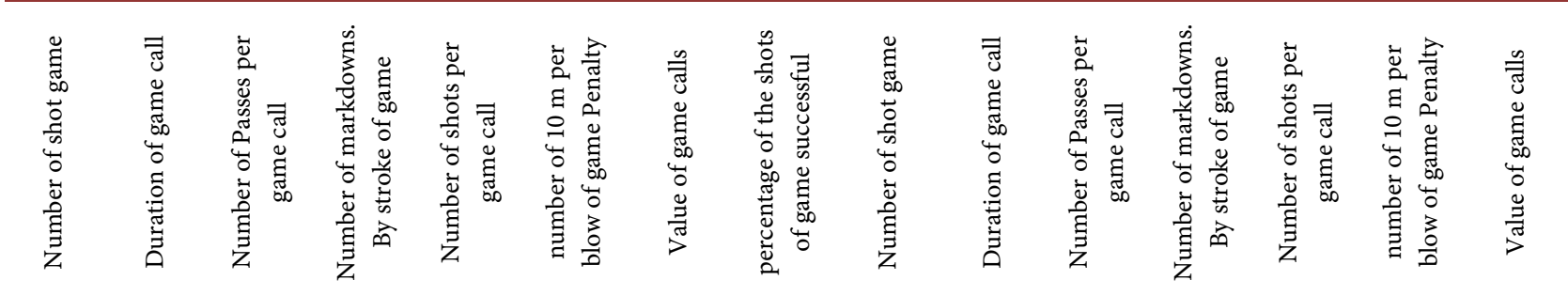

2

3

4

5

6

7

8

9

10

11

12

13

14

15

16

17

18

19

20 